Contents List available at VOLKSON PRESS Intelligent Computing and Information Engineering (ICIE )

DOI : http://doi.org/10.26480/icie.01.2017.84.86

Journal Homepage: : https://www.intelcomp-design.com/

\title{
DESIGN TEMPERATURE AND LIQUID LEVEL CONTROL SYSTEM
}

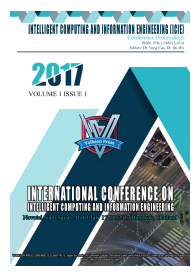

Biqing $\mathrm{Li}^{1}$, Zhao $\mathrm{Li}^{2}$, Liuren Wei ${ }^{1}$

${ }^{1}$ College of Mechanical and Electronic Engineering, Hezhou university, Hezhou Guangxi 542899, China;

${ }^{2}$ Management Engineering Department, Guangxi vocational and technical college of communications, Liuzhou Guangxi 545000, China;

*Corresponding author email: janliful@163.com

This is an open access article distributed under the Creative Commons Attribution License, which permits unrestricted use, distribution, and reproduction in any medium, provided the original work is properly cited

\section{ARTICLE DETAILS}

\section{Article History:}

Received 12 May2017

Accepted 12 July 2017

Available online 14 September 2017

Keywords:

Micro-controller, temperature sereneness driveller IC, relays, electrical bar.

\section{ABSTRACT}

Along long to the continuance is developing of science and technologies for this level to is people's daily is lives keeping improved, so many smart devices location appear on people's daily lives, and this temperature is $0 \mathrm{~K}$, and liquid is leveled controlled is system design for. An important is part to the design do this power oversupply, temperature sensor DS18b20 models, Ultrasonic sensors, LCD1602 model HC-SR04 models of LCD monitors, HK3FF-DC5V-SHG type of relay, heating rods, buzzer, pumps and STC89C52 model of driver ICs. Temperature and liquid very bit control is system designed achieved to feature are to many, respectively has to a contain within liquidizer for the temperature and liquid are bit for heighten achieving real-time to monitored.

\section{OVERALL DESIGN}

The temperature and liquid level control system design of the power part applies the three-terminal voltage regulator IC LM7805 for the system power chip. It has only three pins, output, grounding and input [1-3]. At the same time, using a three-terminal voltage regulator IC to constitute the power supply needs of the external devices is very few, and the circuit also has adjustment tubes and overheating, over-current protection circuits, which use safely and easily, and it is affordable [4]. The LM7805 application circuit diagram is shown in Figure 2.

Working current of pumps: 65MA-500MA; LCD1602 working current of LCD display: 2MA-50MA; Ultrasonic module working current: 15MA; LED working current: 10MA-20MA [5]. The working current of the analysis system with $9 \mathrm{v}$ voltage is less than $1000 \mathrm{ma}$, so the LM7805 three-terminal voltage regulator IC meets the requirements of the design.

\subsection{Ultrasonic Module}

Ultrasonic module performance is very stable, and the monitoring distance is very precise, which can keep the pace with foreign production of advanced technology ultrasonic ranging module [6]. The blind area $(2 \mathrm{~cm})$ of the module is very close and the performance is very stable at the same time, so the accuracy of the ultrasonic module of this model is very high.
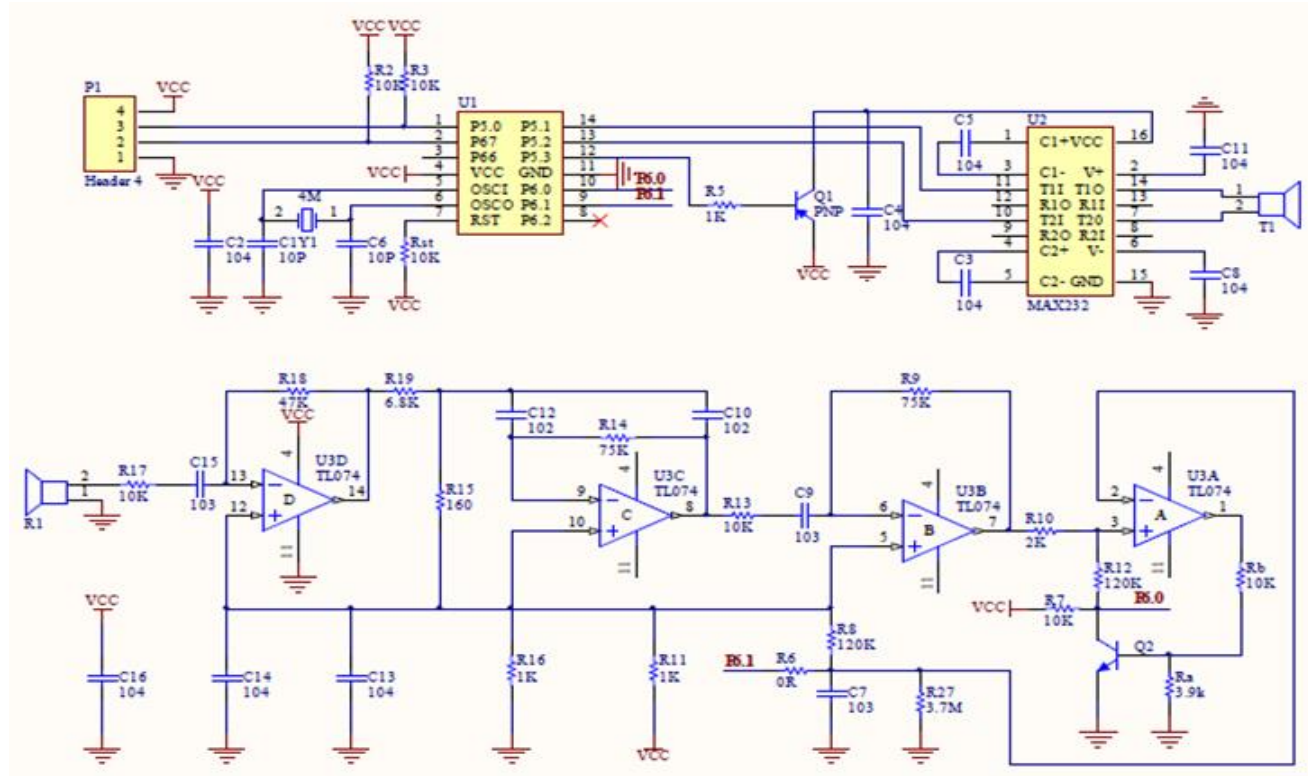

Figure 1: schematic diagram of ultrasonic module 


\section{SYSTEM CIRCUIT DESIGN}

When the program begins, we first have to open the timer, which is used for timing, then the LCD initiates, which prepares for the following LCD display. And then it adds the serial protocol, because DS18B20 is through the serial port to send the data.

After entering the circulating main program, we use MCU I/O to control ultrasonic waves, and first let the ultrasonic wave to emit a sound. At the same time, opening the timer, the program is sent to wait until the ultrasonic module device receives reflected back sound waves, while the timer closes [7]. The time when the sound is sent out and reflected back is the time within the timer, and we know that $340 \mathrm{~m} / \mathrm{s}$ is the speed of the sound in the air. According to the relationship between time and speed, we can calculate the depth of effluent.

Temperature sensor is used to gather the temperature information through the MCU I/O port in the way to the serial communication protocol to the microcontroller, and then the SCM to deal with the decoding of the temperature collected in the digital display on the LCD screen [8].

And the alarm system is the depth of the above and the temperature of real-time detection, and the value of the detection and set the value of comparison [9]. If the detection exceeds our set of scope, then it will conduct the corresponding treatment. The schematic diagram of the hardware design is shown in Figure 1.

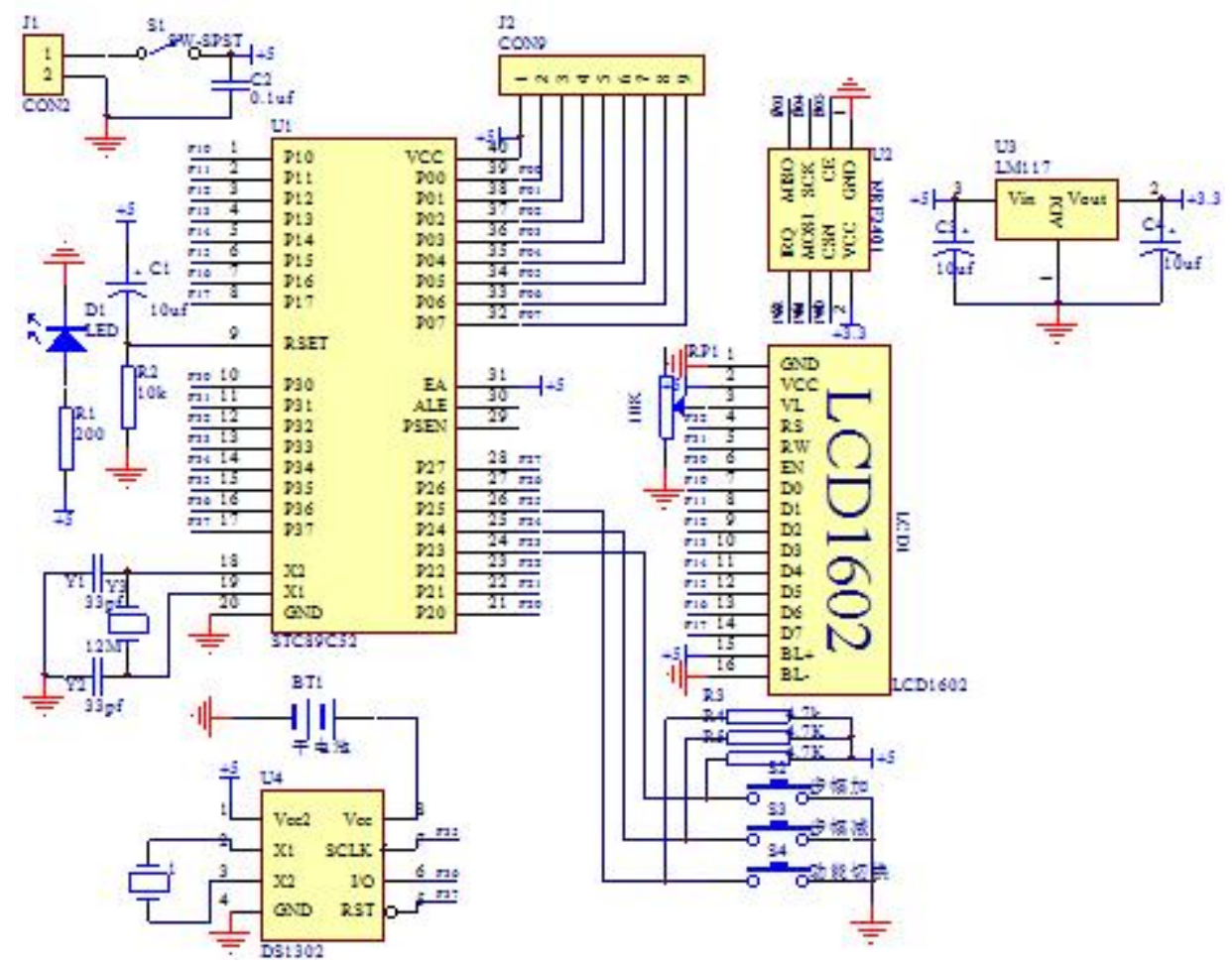

Figure 2: design of temperature and liquid level control system

\section{DEBUGGING ANALYSIS AND SOLUTION}

\subsection{Physical problems and solutions}

After giving the power to the single-chip microcomputer, we found that the single-chip computer cannot work, and we found it is caused by the crystal oscillator, which cannot work properly [10]. The reasons why the crystal oscillator does not work are as follow:

(1) the wiring error of the components in the schematic diagram; (2) It is a monolithic machine, which is not in good quality; (3)The quality of crystal oscillator is not very good; (4) load capacitance or matching capacitance and crystal are mismatching, or the capacitor has the quality problem; (5) PCB board damp leads to the impedance mismatch, so that it cannot be vibrated; (6)the line of the crystal oscillator circuit is unreasonable; (7) there are lines between the pins of the crystal (8)influenced by the peripheral circuit

After the investigation, it is found that it is caused by the mismatching between the capacitance and crystal oscillator, which should be replaced by $30 \mathrm{pf}$ capacitor. After replacing the capacitor, the microcontroller can work.

\subsection{Pumps do not pump and the buzzer not ringing}

When the program is copied into the microcontroller, it is found that when the water level is too high or too low, the pumps are not working and the buzzer does not ring, repeated inspection discovery program, with a multi-meter measurement of the pump and buzzer positive output is only about $2 \mathrm{v}$, by browsing through the information and the Internet to view some relevant knowledge, we found that SCM control uln2003 pin to connect the resistance, because the SCM foot high level is insufficient since the drive uln2003 [11]. After adding 3 of the tensile resistance in the original PCB board, the water can pump and drain normally, and the buzzer can also work properly.

\subsection{Heating rod is not normally heating the liquid}

When the temperature of the liquid in the container is below the minimum alarm value, the heating rod cannot be heated to the liquid after the system emits the acoustic alarm [12]. The cause of this problem is analyzed, which can be divided into the following: (1) The wiring fault of the relay has not been simulated, (2) heating rod sealing agent damage water leakage; (3) the program write error; (4) relay has burned or heated plate burned. After careful examination of the line, we have not found a problem. When using a multi-meter to detect the relay, we found the problem in the relay [13-16]. This is the main reason why I use this model relay. It can use a multi-meter to check whether burned.

\section{SUMMARY}


This temperature and liquid level control system design's advantages are that the monitoring delay error and processing delay error is very small, and when the liquid level too high, too low and it has too high or too low temperature, it can process intelligently and automatically. Its various alarm values can be artificially set, which is very user-friendly and convenient.

This work is supported the following fund:2016 The project of improving the basic ability of young teachers in Colleges and universities in Guangxi:“Design and development of electronic commerce platform of agricultural products based on Semantic Technology"(No,KY2016YB455).2015 college students' innovative training program: "Research on the application of value added travel experience in the mobile terminal of the 'ethnic custom travel' in Guangxi" (No 201511838070);\&\& “The design and development HeYuanTong Campus Mobile Phone APP based on Android" (No 201511838034).Project of scientific research and technology development project of Hezhou: "Design and implementation of agricultural products e-commerce platform based on Semantic Technology" (No,Hekeneng 1506006).

\section{REFERENCE}

[1] Li, B.Q., Ling, Y.F., Zhang, H.Y., Zheng, S.Y. 2016. The Design and Realization of Cherry Tomato Harvesting Robot Based on Iot. International Journal of Online Engineering, 12 (12), 23.

[2] Li, B.Q., Guan, W.L., Zheng, S.Y., Yue, X.G. 2015. Optimisation Design of Corn Precision Seeder Based on Multi-Route and Multi-Channel Control. Journal of The Balkan Tribological Association, 21(4a), 1215.

[3] Zheng, S.Y. 2015. Social Work in Teen Addiction Correction Services Research Under the New Situation, In: Proceedings of the 2015 4th National Conference on Electrical, Electronics and Computer Engineering (Nceece 2015), Xian, China, Pp.252-255.

[4] Li, B.Q. 2016. Intelligent Control Management System and Its Application, In: Proceedings of the 2016 International Conference on Economics and Management Innovations, Wuhan, China, Pp.68-71.

[5] Li, B.Q. 2016. Design and Implementation of Tanks War Game Based on The Android Platform, In: Proceedings Of The 2016 2nd Workshop On Advanced Research And Technology In Industry Applications, Dalian, China, Pp.963-966.

[6] Li, B.Q. 2016. Design of A Tea Garden Anti-Freezing Control System, In: Proceedings Of The 2016 6th International Conference On Machinery, Materials, Environment, Biotechnology And Computer (Mmebc), Tianjin, China, Pp.736-738.

[7] Li, B.Q. 2016. Design of Electronic Compass, In: Proceedings of the 2016 6th International Conference on Machinery, Materials, Environment, Biotechnology And Computer(Mmebc), Tianjin, China, Pp.1240-1243.

[8] Li, B.Q. 2016. Research of Automatically Light-Adjusting Lamp, In: Proceedings of the 2016 International Conference on Computer Engineering, Information Science and Application Technology (Iccia 2016), Guilin, China, Pp.249-252.

[9] Li, B.Q. 2016. The Design and Realization of Fruit Harvesting Robot Based on Iot, In: Proceedings of the 2016 International Conference on Computer Engineering, Information Science and Application Technology (Iccia 2016), Guilin, China, Pp.261-264.

[10] Li, B.Q. 2016. A New Type of Automatic Opening and Closing Light-Operated Curtain, In: Proceedings of the 2016 International Conference on Mechatronics Engineering and Information Technology(Icmeit), Xian, China, Pp.66-69.

[11] Li, B.Q. 2016. Design of The Intelligent Air Humidifier, In: Proceedings of the 2016 International Conference on Mechatronics Engineering and Information Technology (Icmeit), Xian, China, Pp.201-203.

[12] Li, B.Q. 2016. The Design Implementation of The App of Experiencing Guangxi Folk Custom, In: Proceedings of the 2016 International Conference On Economics And Management Innovations, Wuhan, China, Pp.47-50.

[13] Zheng, S.Y. 2015. Digital Display Design of Ethnic Clothing of Nanling, In: Proceedings of the 4th International Conference on Mechatronics, Materials. Chemistry and Computer Engineering 2015(Icmmcce 2015), Xian, China, Pp.2805-2808.

[14] Zheng, S.Y. 2015. Design and Implementation Of Supermarket Personnel Management System Based On Java, In: Proceedings Of The 2015 International Conference On Education, Management And Computing Technology, Tianjin, China, Pp.1724-1727.

[15] Zheng, S.Y. 2016. Analysis Of Internet Of Things Talent Training And Curriculum System Innovation, In: Proceedings Of The 2016 International Conference On Education,Management And Computing Technology(Icemct-16), Hangzhou, China, Pp.957-960.

[16] Zheng, S.Y. 2015. Brief Analysis On "Heyuantong" Campus Mobile Phone App Design, In: Proceedings of The 2015 5th International Conference On Computer Sciences And Automation Engineering, Sanya, China, Pp.151-154 